\title{
A Novel Iterative Detection in Downlink of Massive MIMO System
}

\author{
Mingtong Sun ${ }^{1}$, Jie Wang1, Dongming Wang1, Lijun Zhai ${ }^{2}$ \\ ${ }^{1}$ National Communication Research Laboratory (NCRL), Southeast University, Nanjing, China \\ ${ }^{2}$ CETC 54, Shijiazhuang, China \\ Email: 992021082@qq.com
}

How to cite this paper: Sun, M.T., Wang, J., Wang, D.M. and Zhai, L.J. (2019) A Novel Iterative Detection in Downlink of Massive MIMO System. Journal of Computer and Communications, 7, 116-126. https://doi.org/10.4236/jcc.2019.710011

Received: September 24, 2019

Accepted: October 9, 2019

Published: October 16, 2019

\begin{abstract}
In this paper, we propose the novel iterative detection which operates iteratively on blocks of the received signal in downlink in massive MIMO (Massive Multiple-input Multiple-output) system. This article will combine the equivalent channel with soft detection and soft decoder, and finally propose a new structure for the iterative detection in downlink based on 5G simulation test platform of NCRL and analysis the performance of the novel structure. The simulation result shows iterative algorithm performs better than conventional detection with lower amount of iterations.
\end{abstract}

\section{Keywords}

Iterative Algorithm, Massive MIMO, Novel Structure

\section{Introduction}

$5 \mathrm{G}$ technology has grown and advanced significantly over the years through research and innovation. The key features of $5 \mathrm{G}$ contain high throughput, improved spectrum efficiency reduced latency, better mobility support, and high connection density. To support increased throughput requirements, massive MIMO technology is applied in 5G.

Various techniques like spatial multiplexing, diversity and precoding [1] [2] has supported to improve the channel capacity, energy efficiency, spectral efficiency and the reliability of the communication link. Precoding techniques used in massive MIMO system are to exploit spatial multiplexing gain or diversity gain. It is known that spatial multiplexing is used to increase the spectral efficiency and diversity coding is used to increase the link reliability. In [3] and [4], hybrid precoding and QR methods are introduced respectively. SVD based 
beamforming method to increase the capacity with optimum proper power allocation was proposed by [5] [6]. We use the two-level algorithm to decompose the multi-user MIMO channel into multiple single-user channels, which contains block diagonalization and SVD.

Pilot contamination and interference are well-known problems affecting communication system, requiring the need of an equalizer. Various solutions to the problem were widely investigated. The optimal, iterative equalizer applying soft feedback is LMMSE ISDIC equalizer. Iterative channel estimation and data detection is researched in [7] [8]. We consider time division duplexing since it can exploit channel reciprocity [9]. An innovated double-turbo receiver was proposed to alleviate the pilot contamination and interference, which combines the equivalent channel estimation with the detector and the decoder.

The paper is organized as follows. In Section 2, we propose the system model, including precoding algorithm, detection algorithm and so on. Section 3 introduces the novel detection structure and compares the proposed structure and the traditional one. Some numerical results with a performance comparison are shown in Section 4. Last, Section 5 draws the conclusions on the proposed schemes.

\section{System Model}

The paper considers the downlink of a MU-MIMO system. The cellular scene is a single base station serving K UEs simultaneously. Assume that the uplink and downlink has reciprocity $\left(\boldsymbol{G}_{\mathrm{D}}=\boldsymbol{G}_{\mathrm{U}}^{\mathrm{T}}\right)$. According to [10], the following precoding scheme is used. We first calculate zero force precoding:

$$
\hat{\boldsymbol{G}}=\boldsymbol{G}_{\mathrm{U}}^{*}\left(\boldsymbol{G}_{\mathrm{U}}^{\mathrm{T}} \boldsymbol{G}_{\mathrm{U}}^{*}\right)^{-1}=\left[\begin{array}{lll}
\hat{\boldsymbol{G}}_{1} & \cdots & \hat{\boldsymbol{G}}_{K}
\end{array}\right]
$$

ZF precoding forces the interference of other users to zero.

$$
\boldsymbol{G}_{\mathrm{D}} \hat{\boldsymbol{G}}=\left[\begin{array}{c}
\boldsymbol{G}_{\mathrm{D}, 1} \\
\vdots \\
\boldsymbol{G}_{\mathrm{D}, K}
\end{array}\right]\left[\begin{array}{lll}
\hat{\boldsymbol{G}}_{1} & \cdots & \hat{\boldsymbol{G}}_{K}
\end{array}\right]=\boldsymbol{I}
$$

This means

$$
\boldsymbol{G}_{\mathrm{D}, k} \hat{\boldsymbol{G}}_{j}= \begin{cases}\boldsymbol{I} & k=j \\ \mathbf{0} & k \neq j\end{cases}
$$

MMSE precoding describes the approach which minimizes the mean square error. It does not usually eliminate ISI completely but, minimizes the total power of the noise and ISI components.

$$
\hat{\boldsymbol{G}}=\boldsymbol{G}_{\mathrm{U}}^{*}\left(\boldsymbol{G}_{\mathrm{U}}^{\mathrm{T}} \boldsymbol{G}_{\mathrm{U}}^{*}+\sigma^{2} \boldsymbol{I}_{N_{t} \times K}\right)^{-1}=\left[\begin{array}{lll}
\hat{\boldsymbol{G}}_{1} & \cdots & \hat{\boldsymbol{G}}_{K}
\end{array}\right]
$$

Then make QR decomposition to $\hat{\boldsymbol{G}}_{k}$.

$$
\hat{\boldsymbol{G}}_{k}=\hat{\boldsymbol{Q}}_{k} \hat{\boldsymbol{R}}_{k}
$$

where $\hat{\boldsymbol{Q}}_{k}$ is unitary orthogonal matrix, $\hat{\boldsymbol{R}}_{k}$ is an upper triangular matrix, then 


$$
\boldsymbol{G}_{\mathrm{D}, k} \hat{\boldsymbol{Q}}_{j} \hat{\boldsymbol{R}}_{j}= \begin{cases}\boldsymbol{I} & k=j \\ \mathbf{0} & k \neq j\end{cases}
$$

$\hat{\boldsymbol{Q}}_{k}$ is the precoding matrix which satisfied the principle of BD precoding algorithm. We perform QR decomposition to all the $\hat{\boldsymbol{G}}_{k}$ one by one, then we can get the interference suppression matrix of downlink.

$$
\boldsymbol{W}_{\mathrm{IS}}=\left[\begin{array}{lll}
\hat{\boldsymbol{Q}}_{1} & \cdots & \hat{\boldsymbol{Q}}_{K}
\end{array}\right]
$$

The interference suppression matrix has the ability to blocks diagonalization, the specific property is as follows.

$$
\boldsymbol{G}_{\mathrm{D}} \boldsymbol{W}_{\mathrm{IS}}=\boldsymbol{G}_{\mathrm{U}}^{\mathrm{T}} \boldsymbol{W}_{\mathrm{IS}}=\left[\begin{array}{lll}
\boldsymbol{R}_{1} & & \\
& \ddots & \\
& & \boldsymbol{R}_{K}
\end{array}\right]
$$

where $\boldsymbol{R}_{k} \triangleq \hat{\boldsymbol{R}}_{k}^{-1}=\boldsymbol{G}_{\mathrm{U}, k}^{\mathrm{T}} \boldsymbol{W}_{\mathrm{IS}, k}$. It can be seen that after blocks diagonalization, the interference among multiple users is eliminated. Next, we can make SVD decomposition to matrix $\boldsymbol{R}_{k}$.

$$
\begin{gathered}
\boldsymbol{R}_{k}=\boldsymbol{U}_{k} \boldsymbol{D}_{k} \boldsymbol{V}_{k}^{\mathrm{H}} \\
\boldsymbol{R}_{k}^{\mathrm{T}}=\left(\boldsymbol{U}_{k} \boldsymbol{D}_{k} \boldsymbol{V}_{k}^{\mathrm{H}}\right)^{\mathrm{T}}=\boldsymbol{V}_{k}^{*} \boldsymbol{D}_{k} \boldsymbol{U}_{k}^{\mathrm{T}}
\end{gathered}
$$

From (8)-(9), we can know that the downlink single-user precoding matrix is $\boldsymbol{V}_{k}$ and the uplink single-user precoding matrix is $\boldsymbol{U}_{k}^{*}$. The dimensions of these two matrices are depended on the numbers of streams.

As for detection, [11] proposes a new low-complexity iterative detection for overloaded multiuser MIMO systems. Iterative algorithm of soft interference cancellation is researched in [12] [13] [14]. In this letter, the LMMSE-ISDIC algorithm is used in data detection unit. If we know the priori information of transmitted signal, the mean $\overline{\boldsymbol{s}}$ and variance matrix $\boldsymbol{V}$ of transmitted signal can be calculated. If the priori information is unknown, we can set them to 0 and 1 respectively. The detection value of the kth sending symbol is

$$
\hat{\boldsymbol{s}}_{k}=\boldsymbol{e}_{k}^{\mathrm{H}}-\overline{\boldsymbol{s}}+\boldsymbol{e}_{k}^{\mathrm{H}} \boldsymbol{V} \boldsymbol{H}^{\mathrm{H}}\left[\boldsymbol{H} \boldsymbol{V} \boldsymbol{H}^{\mathrm{H}}+\sigma^{2} \boldsymbol{I}_{\boldsymbol{N}_{\mathrm{r}}}\right]^{-1}(\boldsymbol{r}-\boldsymbol{H} \overline{\boldsymbol{s}})
$$

From the view of downlink receiver, the received signal can be expressed as

$$
\boldsymbol{y}=\boldsymbol{G}_{\mathrm{D}} \boldsymbol{W}_{\mathrm{IS}} \hat{\boldsymbol{V}} \boldsymbol{s}+\boldsymbol{n}
$$

where $\hat{\boldsymbol{V}}$ and $\boldsymbol{s}$ respectively

$$
\begin{gathered}
\hat{\boldsymbol{V}}=\left[\begin{array}{ccc}
\hat{\boldsymbol{V}}_{1} & & \\
& \ddots & \\
& & \hat{\boldsymbol{V}}_{K}
\end{array}\right] \\
\boldsymbol{s}=\left[\begin{array}{c}
\boldsymbol{s}_{1} \\
\vdots \\
\boldsymbol{s}_{K}
\end{array}\right]
\end{gathered}
$$

When combining precoding matrix and channel matrix, we can rewrite (11) as follow, 


$$
\boldsymbol{y}=\boldsymbol{G}_{\mathrm{D}} \boldsymbol{W}_{\mathrm{IS}}\left[\begin{array}{c}
\hat{\boldsymbol{V}}_{1} \boldsymbol{s}_{1} \\
\hat{\boldsymbol{V}}_{K} \boldsymbol{s}_{K}
\end{array}\right]+\boldsymbol{n}=\left[\begin{array}{lll}
\boldsymbol{R}_{1} & & \\
& \ddots & \\
& & \boldsymbol{R}_{K}
\end{array}\right]\left[\begin{array}{c}
\hat{\boldsymbol{V}}_{1} \boldsymbol{s}_{1} \\
\hat{\boldsymbol{V}}_{K} \boldsymbol{s}_{K}
\end{array}\right]+\boldsymbol{n}
$$

Each user received signal is $\boldsymbol{y}_{k}=\boldsymbol{R}_{k} \hat{\boldsymbol{V}}_{k} \boldsymbol{s}_{k}+\boldsymbol{n}_{k}$.

In ideal condition,

$$
\boldsymbol{G}_{\mathrm{D}, k} \boldsymbol{W}_{\mathrm{IS}, k^{\prime}}=\mathbf{0}
$$

However, there are some channel estimation error in realistic scenario, $\boldsymbol{G}_{\mathrm{D}, k} \boldsymbol{W}_{\mathrm{IS}, k^{\prime}} \neq \mathbf{0}$, each user received signal is expressed as

$$
\boldsymbol{y}_{k}=\boldsymbol{G}_{\mathrm{D}, k} \boldsymbol{W}_{\mathrm{IS}, k} \hat{\boldsymbol{V}}_{k} \boldsymbol{s}_{k}+\boldsymbol{G}_{\mathrm{D}, k} \sum_{k^{\prime} \neq k}^{K} \boldsymbol{W}_{\mathrm{IS}, k^{\prime}} \hat{\boldsymbol{V}}_{k^{\prime}} \boldsymbol{s}_{k^{\prime}}+\boldsymbol{n}
$$

Redefining

$$
\begin{gathered}
\tilde{\boldsymbol{y}}_{k}=\widetilde{\boldsymbol{G}}_{k} \boldsymbol{s}_{k}+\tilde{\boldsymbol{n}}_{k} \\
\tilde{\boldsymbol{y}}_{k}=\boldsymbol{y}_{k} \\
\widetilde{\boldsymbol{G}}_{k}=\boldsymbol{G}_{\mathrm{D}, k} \boldsymbol{W}_{\mathrm{IS}, k} \hat{\boldsymbol{V}}_{k} \\
\tilde{\boldsymbol{n}}_{k}=\boldsymbol{G}_{\mathrm{D}, \boldsymbol{k}} \sum_{k^{\prime} \neq k}^{K} \boldsymbol{W}_{\mathrm{IS}, k^{\prime}} \hat{\boldsymbol{V}}_{k^{\prime}} \boldsymbol{s}_{k^{\prime}}+\boldsymbol{n} \\
\boldsymbol{\Xi}_{\boldsymbol{k}} \triangleq \boldsymbol{E}\left(\tilde{\boldsymbol{n}}_{k} \tilde{\boldsymbol{n}}_{k}^{\mathrm{H}}\right)=\sum_{k^{\prime} \neq k}^{K} \breve{\boldsymbol{G}}_{k^{\prime}} \breve{\boldsymbol{G}}_{k^{\prime}}^{\mathrm{H}}+\sigma^{2} \boldsymbol{I}
\end{gathered}
$$

In ideal condition,

$$
\begin{gathered}
\tilde{\boldsymbol{n}}_{k}=\boldsymbol{n} \\
\boldsymbol{\Xi}_{k} \triangleq \mathrm{E}\left(\tilde{\boldsymbol{n}}_{k} \tilde{n}_{k}^{\mathrm{H}}\right)=\sigma^{2} \boldsymbol{I}
\end{gathered}
$$

Defining $\boldsymbol{\Xi}$

$$
\begin{aligned}
\boldsymbol{\Xi} & =\boldsymbol{\Xi}_{k}+\widetilde{\boldsymbol{G}}_{k} \boldsymbol{V} \widetilde{\boldsymbol{G}}_{k}^{\mathrm{H}} \\
& =\sum_{k^{\prime} \neq k}^{K} \breve{\boldsymbol{G}}_{k^{\prime}} \breve{\boldsymbol{G}}_{k^{\prime}}^{\mathrm{H}}+\breve{\boldsymbol{G}}_{k} \breve{\boldsymbol{G}}_{k}^{\mathrm{H}}+\sigma^{2} \boldsymbol{I}-\breve{\boldsymbol{G}}_{k} \breve{\boldsymbol{G}}_{k}^{\mathrm{H}} \\
& =\breve{\boldsymbol{G}}^{\mathrm{H}}+\sigma^{2} \boldsymbol{I}-\breve{\boldsymbol{G}}_{k} \breve{\boldsymbol{G}}_{k}^{\mathrm{H}}
\end{aligned}
$$

So the detected symbol is as follows,

$$
\hat{\boldsymbol{s}}_{k}=\frac{\boldsymbol{e}_{k}^{\mathrm{H}} \widetilde{\boldsymbol{G}}_{k}^{\mathrm{H}} \boldsymbol{\Xi}^{-1}}{1+\left(1-v_{k}\right) \boldsymbol{e}_{k}^{\mathrm{H}} \widetilde{\boldsymbol{G}}_{k}^{\mathrm{H}} \boldsymbol{\Xi}^{-1} \widetilde{\boldsymbol{G}}_{k} \boldsymbol{e}_{k}}\left(\tilde{\boldsymbol{y}}_{k}-\widetilde{\boldsymbol{G}}_{k} \overline{\boldsymbol{s}}+\widetilde{\boldsymbol{G}}_{k} \overline{\boldsymbol{e}}_{k} \overline{\boldsymbol{s}}_{k}\right)
$$

where

$$
\begin{gathered}
\overline{\boldsymbol{s}}=\left\{\begin{array}{cc}
0 & j=k \\
\overline{\boldsymbol{s}}_{j} & j \neq k
\end{array}\right. \\
{[\boldsymbol{V}]_{j, j}= \begin{cases}1 & j=k \\
v_{j} & j \neq k\end{cases} }
\end{gathered}
$$

The mean and variance of sending signals can be calculated by 


$$
\begin{gathered}
\overline{\boldsymbol{s}}_{k}=\mathrm{E}\left(\boldsymbol{s}_{k} \mid \hat{\boldsymbol{s}}_{k}\right)=\sum_{\boldsymbol{s}_{k} \in \boldsymbol{S}} \boldsymbol{s}_{k} \mathrm{P}\left[\boldsymbol{s}_{k} \mid \hat{\boldsymbol{s}}_{k}\right] \\
v_{k}=\sum_{\boldsymbol{s}_{k} \in \boldsymbol{S}}\left|\boldsymbol{s}_{k}\right|^{2} \mathrm{P}\left[\boldsymbol{s}_{k} \mid \hat{\boldsymbol{s}}_{k}\right]-\left|\overline{\boldsymbol{s}}_{k}\right|^{2}
\end{gathered}
$$

where $\boldsymbol{S}$ is the set of modulated symbols.

\section{Receiver Structure}

\subsection{Traditional Receiver Structure}

In the traditional receiver structure, there is no information interaction between the detection unit, the decoder unit and the equivalent channel unit (Figure 1).

\subsection{Proposed Receiver Structure}

In fact, the extra information obtained by the decoder unit can facilitate the equivalent channel unit so as to eliminate the estimation error and improve the performance of data detection. The same idea is applied between channel estimation and data detection in [15] [16]. The overall framework of the proposed receiver structure is as Figure 2.

\subsubsection{Equivalent Channel Unit}

As Figure 2 clarifies, receiver firstly obtains the equivalent channel value at the pilot point by sending the frequency-domain pilot sequence and uses a certain interpolation algorithm to obtain initial equivalent channel value at the entire frequency point. The equivalent channel matrix is obtained by the product of the channel matrix and multi-level precoding matrix. It needs to note that the frequency-domain pilot sequence is the value of multi-level precoding matrix. The equivalent channel matrix is then used for detection unit.

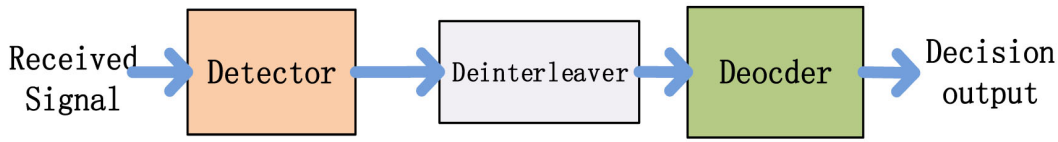

Figure 1. Traditional receiver structure.

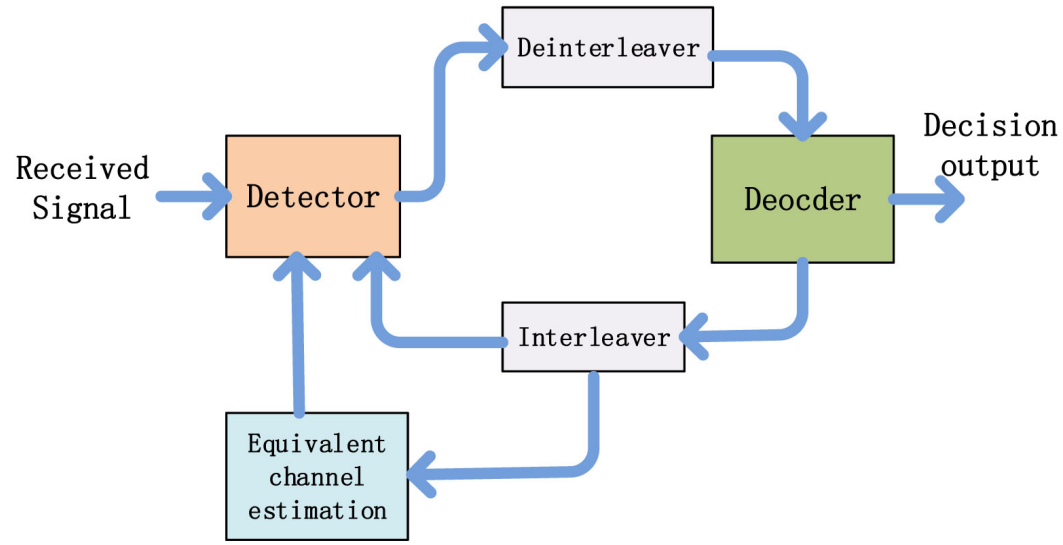

Figure 2. Proposed receiver structure. 
When data has already been processed by the decoder unit, the judged symbols can be sent back to the equivalent channel estimation unit. The channel estimation unit can use the received signal and the reconstructed signal to re-estimate the channel matrix. The reconstructed signal is acquired by the product of the judged symbols and multi-level precoding matrix. In this paper, we use Least-Square algorithm to estimate the equivalent channel. LS algorithm is described by the following formula.

$$
\widehat{\boldsymbol{H}}_{\mathrm{LS}}=\left(\boldsymbol{s}^{\mathrm{H}} \boldsymbol{s}\right)^{-1} \boldsymbol{s}^{\mathrm{H}} \boldsymbol{y}
$$

where $s$ is the reconstructed signal and $\boldsymbol{y}$ is the corresponding received sequence.

\subsubsection{Detection Unit}

The detection unit has accepted the LMMSE-ISDIC algorithm to detect the received symbols. The LMMSE-ISDIC algorithm performs a matrix inversion operation in every single iteration. The complexity of LMMSE-ISDIC in single iteration is relatively low. LMMSE-ISDIC is an iterative algorithm and need to calculate symbolic probability, prior mean and variance (Figure 3).

\subsubsection{Decoder Unit}

Turbo decoder algorithm is applied in the decoder unit. In the iterative detection algorithm, the extrinsic information of decoder will be feed back to the detection unit as the priori information. As the information exchange among the detection unit, the decoder unit and the equivalent channel estimation units, the precision of the three units are enhanced simultaneously during the overall iteration process, which is similar to turbo structure.

\section{Simulation}

The simulation environment of this paper is similar to SCME Urban micro-cell, it has six clusters, each cluster delay and power are in Table 1.

The paper considers one base station in one cell and the base station serves 16 UEs simultaneously, each UE with 8 antennas. Correspondingly, the base station has 128 antennas. The number of each UE's data stream is 4 . The modulation type is QPSK. Assuming that users are evenly distributed around the base station.

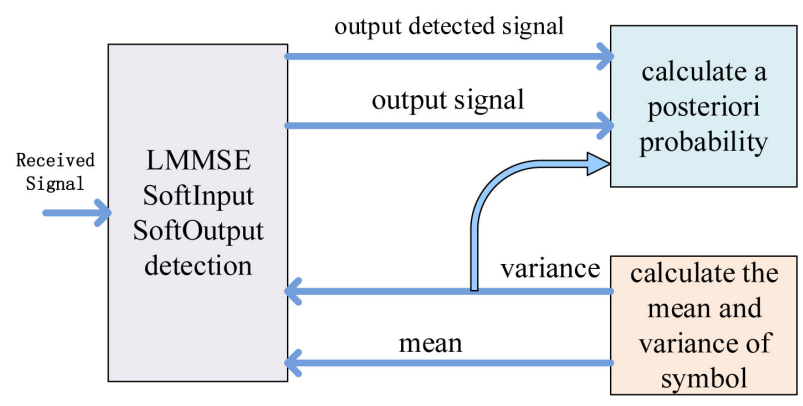

Figure 3. LMMSE-ISDIC structure. 
Table 1. Cluster delat and power.

\begin{tabular}{ccc}
\hline Cluster\# & Delays $[\mathrm{Ns}]$ & Power $[\mathrm{dB}]$ \\
\hline 1 & 0 & 0 \\
2 & 200 & -29.05 \\
3 & 287.5 & -3.57 \\
4 & 662.5 & -20.94 \\
5 & 812.5 & -5.28 \\
6 & 925 & -26.96
\end{tabular}

The article gives the BER (Bit Error Rate) and system achieve rate in downlink when the speed of UE is $3 \mathrm{~km} / \mathrm{h}$. The achieve rate is calculated by data blocks, each data block containing 2400 information bits.

\subsection{LMMSE Iterative Detector}

In order to work out the performance of the LMMSE iterative detector, we set the number of small iteration respectively. Need to explain that in the simulation the small iteration corresponds to the number of LMMSE iteration and the big iteration corresponds to the number of the process of the detector, decoder and equivalent channel.

In Figure 4, there is no decoder and no overall iteration and only LMMSE iteration, which means the big number is zero and the small number is $0,1,2,3$, 4, 5. The number zero means no iteration in LMMSE detector. From Figure 4, there is remarkable improvement in performance. As the number gradually increases one by one, the performance rises little by little.

\subsection{Big Iteration}

To figure out the performance of the big iteration between equivalent channel estimation unit, detection unit and decoder unit, we set the small iterative number to zero. The big iterative number is set to $0,1,2,3$ respectively. The number zero means no information among these three separate units.

From Figure 5, we can learn that the performance improves gradually as the big number increases one by one. The big number 2 improves $0.7 \mathrm{~dB}$ approximately compared to the big number 0 in bit error rate at $10^{-4}$. To reduce the complexity, it is appropriate to set the big number to 2 .

Combining big iteration with LMMSE iterative detector, we could achieve much better performance. To achieve the best effect for application, the number of the LMMSE iteration is set to 3 , and the number of the big iteration is set to 2 , which could make tradeoffs between complexity and performance.

The LMMSE-ISDIC detection algorithm has to do a matrix inversion operation in each iteration. The complexity of the algorithm is approximately $O\left(N_{t}^{3}\right)$. It also needs to calculate symbolic probability, prior mean and variance. However, the complexity maintains at $O\left(N_{t}^{3}\right)$, suitable for hardware implementation. 


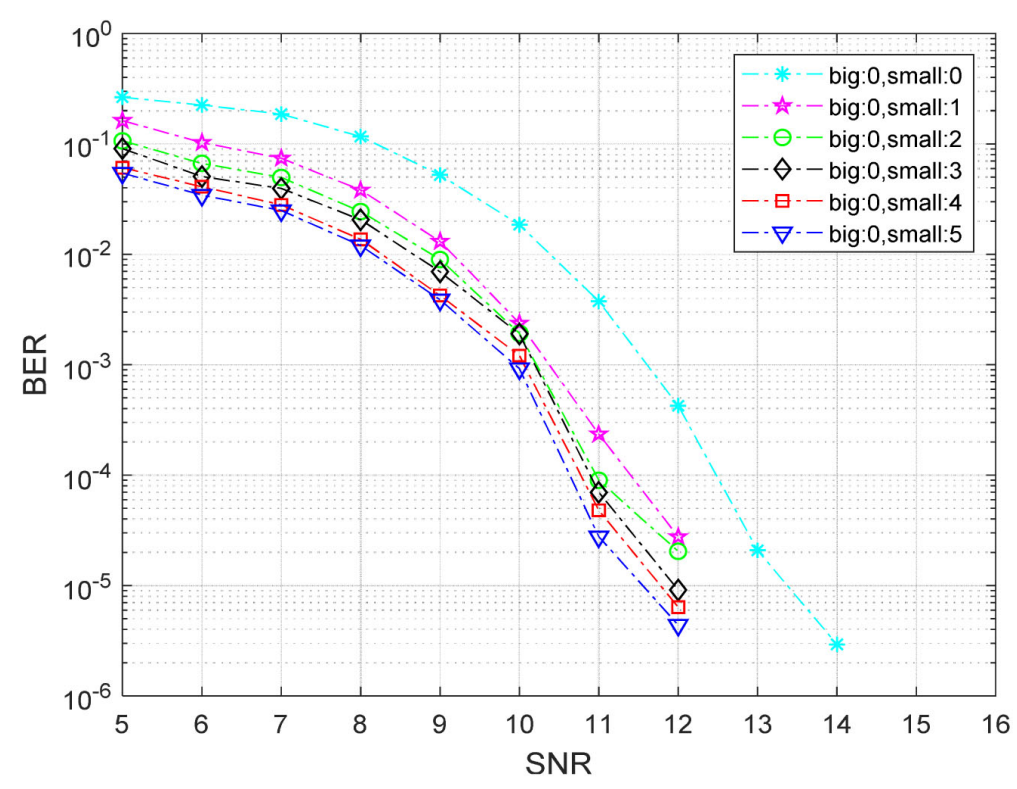

Figure 4. Bit error rate.

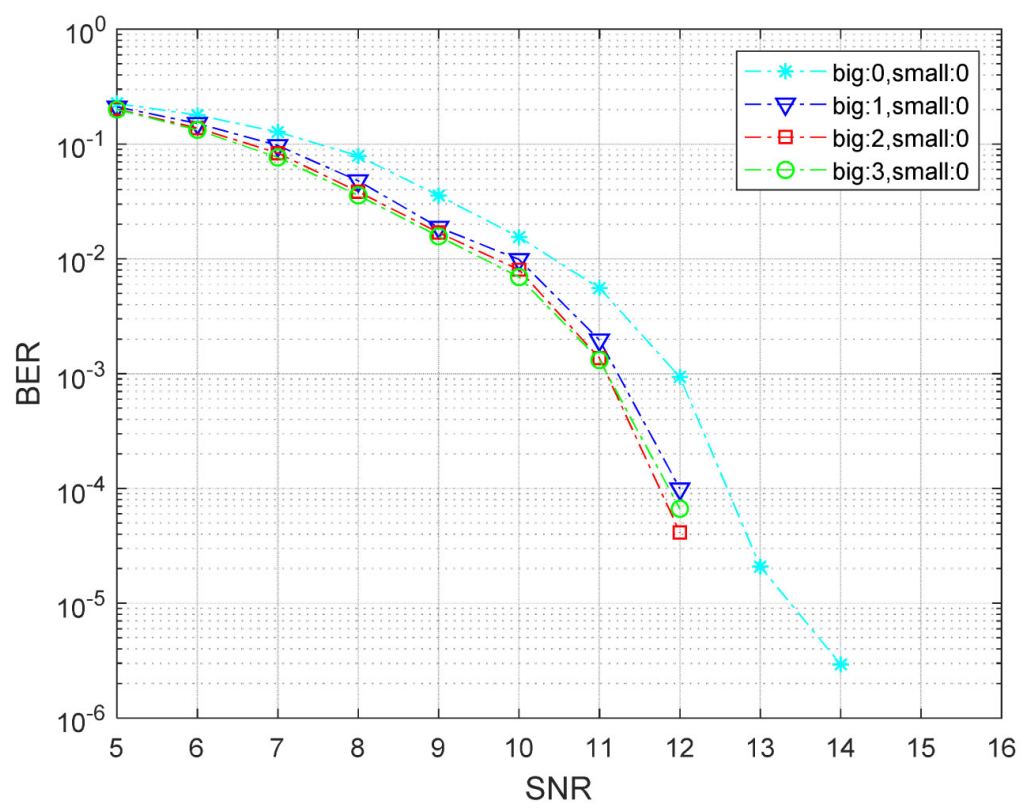

Figure 5. Bit error rate.

\subsection{Comparison}

To enhance the performance better, the big iteration and small iteration are combined together and run at once. In the meantime, we also perform ZF detector and LMMSE detector.

From Figure 6, the performance is enhanced by the overall iteration. Obviously, the big number 2 and small number 3 can improve $2.3 \mathrm{~dB}$ in bit error rate at $10^{-5}$ compared to LMMSE detector. The performance between $\mathrm{ZF}$ and LMMSE is similar. In Figure 7, the former reaches saturation of achievable rate faster than the latter by about $2.3 \mathrm{~dB}$. 


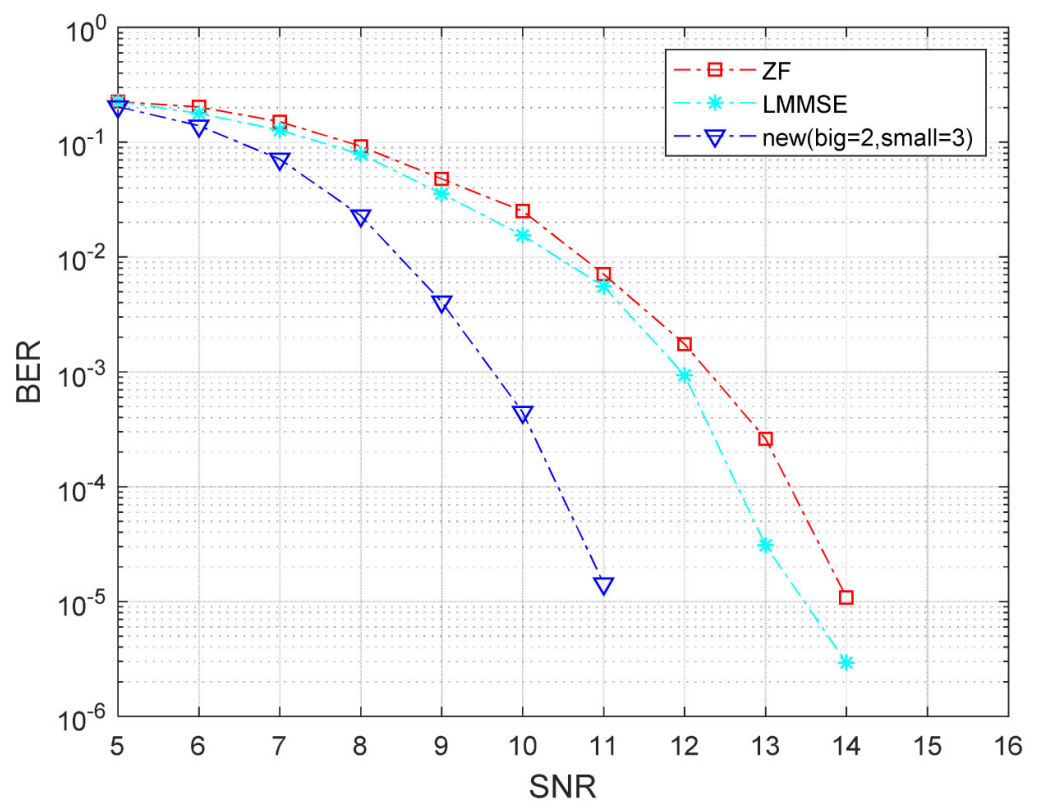

Figure 6. Bit error rate.

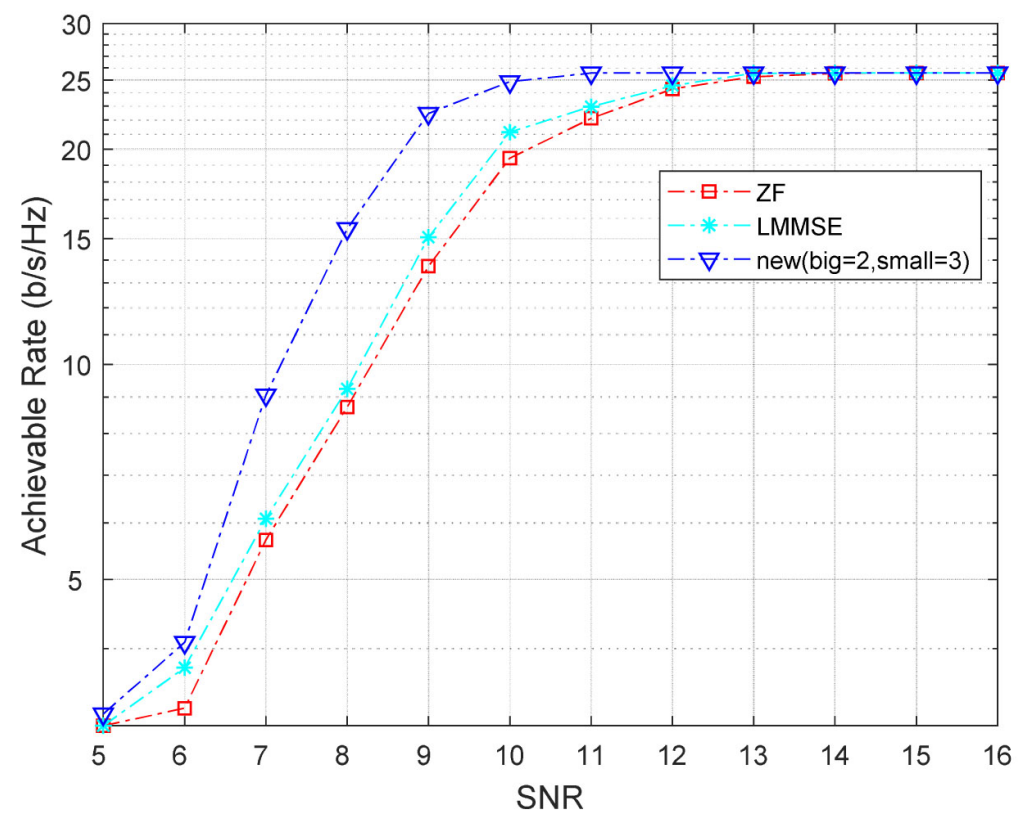

Figure 7. System achieve rate.

\section{Conclusion}

From these simulation results, the overall iteration between equivalent channel estimation unit, detection unit and decoder unit can greatly improve system performance at the cost of complexity since it can exchange information among different units. To reduce system complexity, we can set the number of overall iteration to a relatively small number, which can still create a good performance gain. The simulation result has showed that the proposed double-turbo iterative receiver structure can enhance the performance of whole system. 


\section{Conflicts of Interest}

The authors declare no conflicts of interest regarding the publication of this paper.

\section{References}

[1] Pradhan, B.B. and Roy, L.P. (2014) MIMO Beamforming in Spatially and Temporally Correlated Channel. Proc. IEEE India Conference (INDI-CON), December 2014, 1-5. https://doi.org/10.1109/indicon.2014.7030636

[2] Zheng, L. and Tse, D.N.C. (2003) Diversity and Multiplexing: A Fundamental Tradeoff in Multiple-Antenna Channels. IEEE Transactions on Information Theory, 49, 1073-1096. https://doi.org/10.1109/tit.2003.810646

[3] Ahmed, I., Khammari, H. and Shahid, A. (2017) Resource Allocation for Transmit Hybrid Beamforming in Decoupled Millimeter Wave Multiuser-MIMO Downlink. Access IEEE, 5, 170-182. https://doi.org/10.1109/access.2016.2634096

[4] Huang, Z.Y. and Tsai, P.Y. (2011) Efficient Implementation of QR Decomposition for Gigabit MIMO-OFDM Systems. IEEE Transactions on Circuits and Systems I: Regular Papers, 58, 2531-2542. https://doi.org/10.1109/tcsi.2011.2123770

[5] Zhan, C.-Z., Jheng, K.-Y., Chen, Y.-L., Jheng, T.-J. and Wu, A.-Y. (2009) High-Convergence-Speed Low-Computation-Complexity SVD Algorithm for MIMO-OFDM Systems. Proc. International Symposium on VLSI Design Automation and Test, April 2009, 195-198. https://doi.org/10.1109/vdat.2009.5158128

[6] Tsai, P.Y. and Liu, C.Y. (2015) Reduced-Complexity SVD with adjustable Accuracy for Precoding in Large-Scale MIMO Systems. Proc. IEEE Workshop on Signal Processing Systems, October 2015, 1-6. https://doi.org/10.1109/sips.2015.7345023

[7] Rezk, M. and Friedlander, B. (2008) Joint Channel-Symbol Estimation for High-Performance Differential MIMO. Signals Systems and Computers 2008 42nd Asilomar Conference, 1744-1748. https://doi.org/10.1109/acssc.2008.5074725

[8] Wu, C.-L., Skoglund, M., Chen, P.-N. and Han, Y.S. (2009) A Systematic Space-Time Code Design and Its Maximum-Likelihood Decoding for Combined Channel Estimation and Error Correction. IEEE International Symposium, 764-768. https://doi.org/10.1109/isit.2009.5205641

[9] Wang, D.-M. (2015) 5G Test Platform Algorithm and Principle from NCRL (National Mobile Communication Research Lab, Southeast University).

[10] Sung, H. (2009) Generalized Channel Inversion Methods for Multiuser MIMO Systems. TCOM, 2009.

[11] Chen, M. and Burr, A.G. (2013) Low-Complexity Channel Selection and Iterative Detection for Overloaded Uplink Multiuser MIMO OFDM System. 2013 IEEE 77 th Vehicular Technology Conference, 1-5. https://doi.org/10.1109/vtcspring.2013.6692621

[12] Gerstacker, W.H., Ralfr, M. and Huber, J.B. (2000) Iterative Equalization with Adaptive Soft Feedback. IEEE Trans. on Commun., 48, 1462-1466. https://doi.org/10.1109/26.870012

[13] Yang, T.-Q., Wang, J., Wang, D.M. and Zhai, L.J. (2018) Joint Detection in Uplink of Massive MIMO System. IEEE MTT-S International Wireless Symposium, May 2018. https://doi.org/10.1109/ieee-iws.2018.8400958

[14] Douillard, C., Jézéquel, M. and Berrou, C. (1995) Iterative Correction of Intersymbol Interference: Turbo-Equalization. European Transactions on Telecommunica- 
tions, 6-5, September 1995, 507-511. https://doi.org/10.1002/ett.4460060506

[15] Stoica, P., Vikalo, H. and Hassibi, B. (2003) On Joint ML Channel Estimation and Signal Detection for SIMO Channels. Proc. IEEE ICASSP, 4 Apr 2003, 13-16. https://doi.org/10.1109/icassp.2003.1202529

[16] Vikalo, H., Hassibi, B. and Kailath, T. (2004) Iterative Decoding for MIMO Channels via Modified Sphere Decoding. IEEE Trans. Wireless Commun, 3-6 November 2004, 2299-2311. https://doi.org/10.1109/twc.2004.837271 